\title{
Multidomain IT Architectures for Next-Generation Communications Service Providers
}

\author{
Rob Brennan, Kevin Feeney, John Keeney, and Declan O'Sullivan, Trinity College Dublin \\ Joel J. Fleck II, Hewlett-Packard
}

Simon Foley, University College Cork

Sven van der Meer, Waterford Institute of Technology

\begin{abstract}
Enabling interdomain and end-to-end management are major challenges for IT architectures supporting agile next-generation communications service providers. This requires explicit management of the interdomain relationships themselves rather than treating extradomain resources or services as equivalent to internal capabilities. In this article we describe a general layered model for describing interdomain relationships and a concrete architecture for a domain relationship manager based on a combination of model-driven development and semantic web technology. Our prototyping efforts are discussed, and both examples and descriptions aid gaining an understanding of the underlying technologies. Finally, a use case is presented to illustrate the application of these techniques and especially to show the dynamic behavior of a system based on this engineering approach.
\end{abstract}

\section{Challenges for Next- Generation Service Providers}

The boom in Internet-based services has challenged traditional communication service providers (CSPs) as they strive to avoid becoming purveyors of commodity bit pipes. CSPs are seeking to leverage their customer knowledge, enterprise-quality services, billing, and security infrastructures to widen their customer and revenue base. The agility and adaptability of CSPs' service delivery platforms (SDPs), in terms of their support for new business models, and ability to selectively open the network and engage in value networks with customers and suppliers are widely seen as keys to CSPs achieving their full business potential.

This trend, along with information and communications technology convergence, has led to renewed interest in the application of proven IT technologies to CSP SDPs and operations, administration, and maintenance (OAM) infrastructures. IT technologies present two challenges for CSPs: to support the traditional stringent real-time and scalability requirements, and to provide the required flexibility needed for distributed multivendor environments. For example, one popular IT best practice service governance model is the IT Infrastructure Library (ITIL) [1]. ITIL version 3 offers a comprehensive approach to governing the creation, design, development, deployment, operation, change management, and eventual termination of services. It has also been considerably reworked to directly reference service-oriented architecture (SOA) concepts, whereby combining the strong governance model in ITIL with the flexibility of SOA, CSPs can build adaptable, value-driven, reusable systems. However, how can this process-centric approach be applicable to the fractured business models and proliferation of multi-enterprise value chains based on virtual operators, software as a service, and cloud-based solutions in the CSP space? Dictatorial, end-toend, process-based management systems that require unfettered control and reliable global knowledge are of limited use in the dynamic multi-organizational environments that are becoming the norm for CSPs. Similarly, modern trends in OAM have stressed the deployment of autonomic and adaptive systems in order to reduce operational expense (OPEX) and increase flexibility, but current work has largely focused on centralized architectures and singleCSP control systems.

Ignoring interdomain systems management and the organizational complexity of modern enterprises means that no matter how adaptive or autonomic an application (and the network it runs on) is, it is still basically a static application unless the network sensing, analysis, and control systems can adapt with the multidomain managed system as its solutions and applications reconfigure. To do this without explicitly and 


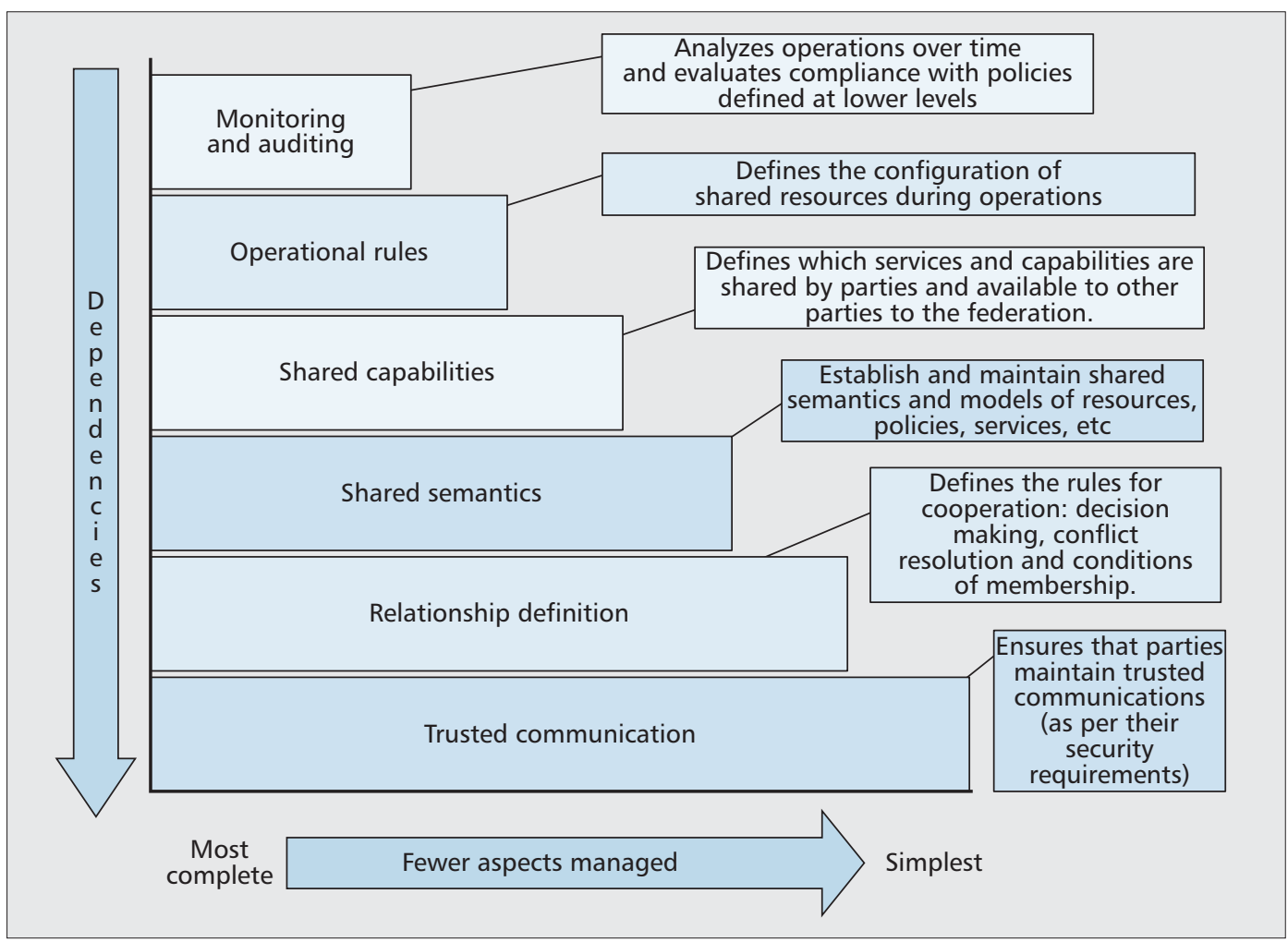

Two important
aspects of a
relationship model
are modeling shared
capabilities and the
specification of the
operational rules that
govern the use of
those capabilities.
These are both
well-known features
of traditional
OAM models.

Figure 1. Layered relationship mode (LRM).

dynamically managing the relationships between the domains contributing to the communications service delivery is ineffective. In addition, the inherent and incurable heterogeneity of interdomain systems means that the network and service descriptions used for management must be extended beyond the static semantic description of their components. Thus, we identify multidomain relationship management based on semantic (knowledge) models as crucial to the deployment of IT infrastructure supporting nextgeneration networks in CSPs.

\section{A CONCEPTUAL MOdEL FOR RELATIONSHIP MANAGEMENT}

Having identified the importance of managing relationships and organizational diversity for the ultimate success of flexible IT systems supporting modern CSPs, we now explore the question of how this can be achieved.

\section{Definition of Domains}

Before discussing domain relationships, it is important to first define what we mean by domains. Here, a domain (or management domain) is defined as an autonomous (self-governing) administrative entity that has a specific business role, and consequent internal goals and local policies [2]. It has a clear boundary defined by the scope of its authority over resources or artefacts. Example domains are business units (e.g., customer billing) within an organization or whole enterprises, such as a third-party customer relations management (CRM) company.

However, domains do not exist in a vacuum. In order to deliver useful services and business value, they often cooperate with or use the capabilities of other domains. These relationships with other domains must themselves be managed: this requires an explicit model of the relationships. Two important aspects of a relationship model are modeling shared capabilities and the specification of the operational rules that govern the use of those capabilities. These are both well-known features of traditional OAM models. Unlike traditional OAM systems, multidomain systems lack a central authority guaranteeing interoperability and need:

- To define or negotiate both shared semantics and a set of protocols for decision making, conflict resolution, and relationship membership

- Trust management to enable domains to communicate with an appropriate level of information security and guarantees of relationship integrity

- Relationship auditing and monitoring to provide evidence of long-term conformance to the agreed parameters of a relationship

\section{A LAYERED ReLATIONSHIP MOdel}

In this work the term domain relationship is employed to describe cross-organizational capability sharing agreements. However, organizational arrangements between autonomous entities vary widely in scope, and can be complex and multifaceted. Thus, models of relationships must be capable of capturing and reflecting the most important factors that vary across such arrangements if they are to support modeling the evolving, dynamic nature of the real world. These relationships can be hierarchical, whereby we call them domain compositions, or peer-to-peer, whereby we designate them domain federations. 


The layers represent
the most important
aspects of cross-
organizational rela-
tionships for
successful persistent
organizational rela-
tionships, with their
relative positioning in
the layered model
representing the
dependencies
between the ele-
ments that constitute
such an agreement.

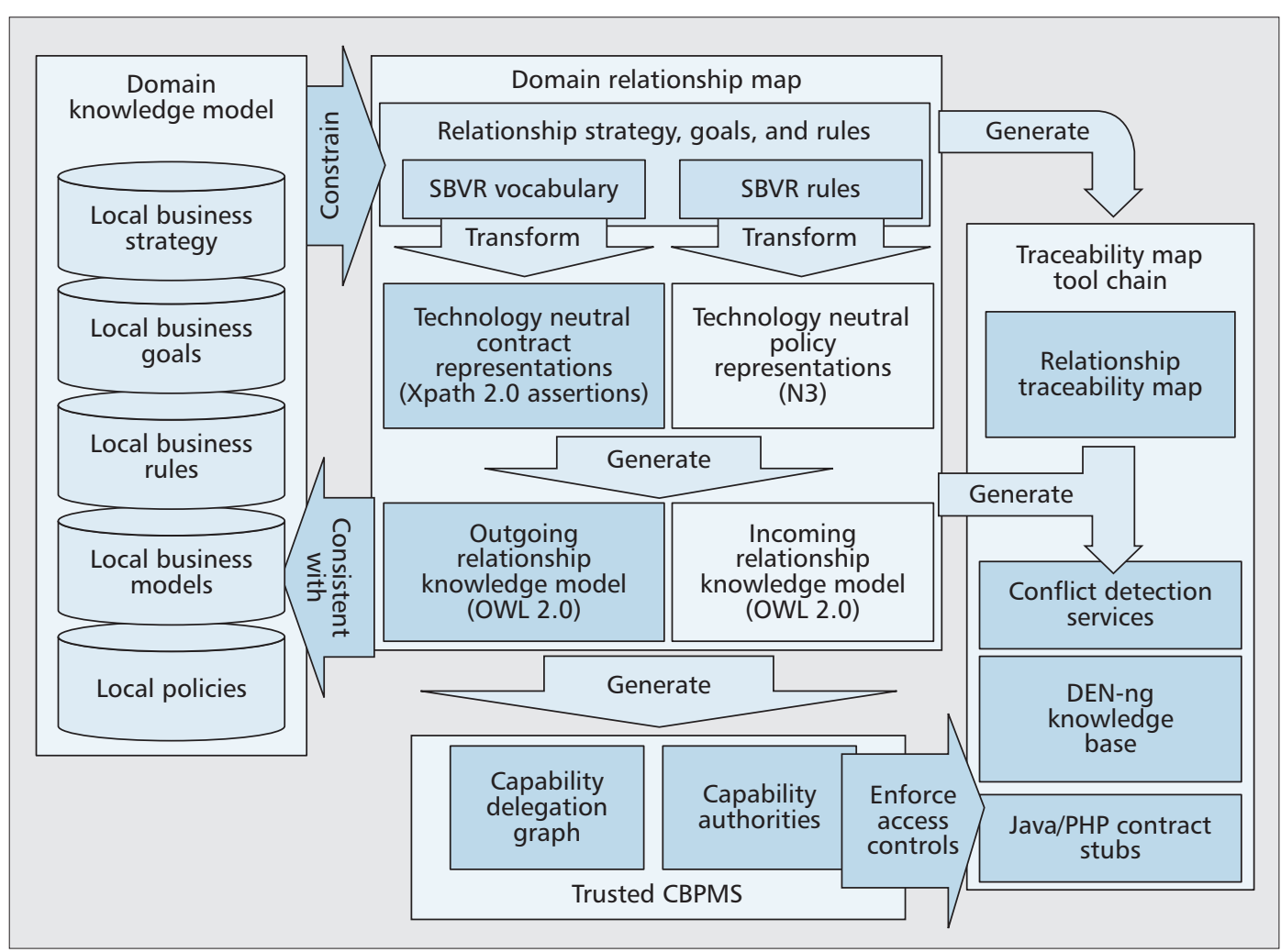

Figure 2. Domain relationship manager architecture.

The Layered Relationship Model (LRM) (Fig. 1), is a general-purpose conceptual model of the components of a domain relationship. The model is decomposed into layers, with each layer representing one aspect of the organizational arrangement. This layered model should not be confused with a communications stack - in some relationships there may be cross-layer interactions, and layers may be empty. Its main purpose is to serve as a useful model for decomposing relationships in order to render their definition and maintenance more tractable and transparent. The layers represent the most important aspects of cross-organizational relationships for successful persistent organizational relationships, with their relative positioning in the layered model representing the dependencies between the elements that constitute such an agreement. In the next section we deal with concrete mechanisms for instantiating this model.

\section{A Multidomain Relationship MANAGEMENT ARCHITECTURE}

This section describes an architecture for multidomain IT systems relationship management based on three key components:

- The domain relationship map (DRM): An explicit model of the relationship from an individual domain's perspective. It supports aspects of LRM operational rules, shared capabilities, shared semantics, and relationship definition layers.

- The trusted community-based policy management system (TCBPMS): A secure, dis- tributed model of multidomain relationship structures and shared capability authorities (access controls or other operational rules). It supports LRM shared capabilities and distributed operational rules. It also includes an infrastructure for distributed trust management that supports the LRM trusted communication layer.

- A relationship traceability map (TM) tool chain: To support relationship context tracking, contract-policy analysis, and contract stub code generation.

The relationships are coordinated and managed by peering, context-aware domain relationship managers (Fig. 2). The domain relationship and traceability maps described below are core tools facilitating this adaptive management. The use of technology-neutral models allows management systems to verify semantic consistency across the relationship using ontologic reasoning. The use of ontologies in conjunction with representations of business goals, policies, and associations allows reasoning about the compatibility or consistency of proposed or existing relationships. The use of technology neutral models also facilitates development of implementations of each side of a relationship using an approach based on model-driven architecture (MDA), reducing handcrafting and testing.

The TCBPMS provides support for integration with the security infrastructure and enforces distributed access controls on interdomain contracts (service interfaces), but also supports distributed, secure formation and management of the relationships between the domains. The subsections below describe these components and the generated artifacts in more detail. 
The domain relationship manager is also responsible for coordinating with its peers from other domains to decide when to mediate, accept or terminate relationships. It needs to handle both domain composition and federation. In the case of composition, both the external ontologies and the external relationship maps must be consistent. If any inconsistencies exist, either the management system must mediate and resolve them, or the relationship must be abandoned. Failure to achieve consistency across the relationship would amount to domains within an organization working toward different, potentially conflicting goals.

Federations only require the ontologies of the participating domains to be consistent, while the goals, policies, and associations of the DRM must be compatible. Incompatibilities between federated domains trigger either a negotiation phase between relationship managers to resolve the incompatibilities, or abandoning the federation to search for a replacement domain satisfying the consistency and compatibility requirements.

\section{THE DRM}

To dynamically create, modify, and remove relationship agreements between domains, we introduce the DRM [3] concept, which models each side of the relationship. It describes, based on the knowledge model of the exposing domain (describing internal business strategy, business goals, business rules, local policies, and resources), an ontology-based knowledge model for exported capabilities, the goals of the potential relationship, and the policies governing it. Not only must the relationship's ontology (knowledge model) be consistent with (and a subset of) the internal ontology of the domain, but the business strategy, goals, and rules of the relationship must also be consistent with those of the domain.

These models are used in two ways:

- To manage the lifecycle of the relationship by ensuring:

-That the model on each side of the relationship remains consistent with the internal model of its associated domain

-That both models on each side of the relationship remain compatible (or consistent - depending on what type of relationship is implemented) with the model on the other side of the relationship

- As the basis for a model-driven approach for deriving and producing the technologyspecific software artifacts that implement the relationship

The business goals of the relationship are documented in Semantics of Business Vocabulary and Rules (SBVR) (described below) in two parts: a vocabulary consistent with the ontology of the relationship; and SBVR rules, which must be consistent with the business rules of the domain. The SBVR vocabulary and rules describing the relationship are used to develop the technology-neutral descriptions of the policies and capabilities of the relationship using model-driven transformations. The fact types of the SBVR vocabulary are used as the basis for a transformation to the XPATH 2.0 assertions that describe the capabilities desired or offered by the relationship. The SBVR rules are used as the input for a transformation to the technologyneutral Notation3 (N3) representation of the policies governing the operation and management of the relationship.

Key Technologies for Relationship Modeling - Many of the technologies deployed in the DRM are not well known by non-knowledge engineers, so we present brief overviews here.

MDA - The Object Management Group (OMG) has defined the MDA approach as a methodology for developing distributed applications by defining a computational-independent business model (CIM), which is transformed to a platform-independent base model (PIM) and to one or more platform-specific models (PSMs) and interface definition sets. Using this approach, neither the CIM nor the base PIM describing the application behavior needs to be changed to support modifications in implementation technologies. The model-driven approach described in this article extends this approach by using transformations to provide tools to validate consistency of interdomain relationships with the internal specifications of the participating domains, and using transformations to generate both policies governing the relationship, the code implementing the relationship and the tools needed to monitor and maintain its health.

Resource Description Framework and Web Ontology Language - The Resource Description Framework (RDF) is the World Wide Web Consortium (W3C) standard for meta-data [4]. It is based on making three-part entity-attribute-value assertions called triples that can be combined into a directed graphbased data, information, or knowledge representation. The base RDF specification has no inherent typing mechanisms until it is extended with the RDF Schema (RDFS) specification. RDF/RDFS are especially useful for flexibly representing data about entities that have a very large range of potential attributes (e.g., if the attributes are unknown), such as when they are defined across a domain boundary where alien concepts may be modeled or common concepts may be modeled in unfamiliar ways. The second property of the RDF that is especially useful in cross-domain modeling is the ability to easily merge data about a single entity from multiple sources.

The Web Ontology Language (OWL) [5] is a set of semantic web representation languages designed to capture ontological concepts, instances of concepts and relationships. OWL builds extensively on RDF/RDFS, but provides additional vocabulary and semantics to better capture the meaning of concepts, relationships, and instances. OWL ontologies are commonly made available in RDF/XML format. One of the main advantages of OWL's formal semantics over other ontological representation approaches is the ability to use automatic inference engines to extract additional semantic statements that were implicit in the ontology and make them directly accessible.
Incompatibilities

between Federated

Domains trigger

either a negotiation

phase between Rela-

tionship Managers to

resolve the

incompatibilities or

abandoning the

Federation to search

for a replacement

Domain satisfying

the consistency and

compatibility

requirements. 


\begin{tabular}{|c|c|}
\hline \multicolumn{2}{|l|}{ Consumer } \\
\hline Definition: & person or resource proxy \\
\hline \multicolumn{2}{|l|}{ Customer } \\
\hline Definition: & entity that pays for goods or services \\
\hline Synonym: & client \\
\hline General concept: & Consumer \\
\hline \multicolumn{2}{|l|}{ Customer type: } \\
\hline \multirow[t]{2}{*}{ Definition: } & concept that specifies the Customer and that classifies \\
\hline & a Customer based on a Service Level \\
\hline Concept type: & categorization type \\
\hline \multicolumn{2}{|l|}{ Platinum customer } \\
\hline Concept type: & Customer type \\
\hline Definition: & Customer who is charged 5 per unit \\
\hline \multicolumn{2}{|l|}{ Gold customer } \\
\hline Concept type: & Customer type \\
\hline Definition: & Customer who is charged 2 per unit \\
\hline \multicolumn{2}{|l|}{ Silver customer } \\
\hline Concept type: & Customer type \\
\hline Definition: & Customer who is charged 1 per unit \\
\hline \multicolumn{2}{|c|}{$\begin{array}{l}\text { Rule 8: It is obligatory that the Rater assign a Customer type to a } \\
\text { Normalized Usage Record }\end{array}$} \\
\hline \multicolumn{2}{|c|}{$\begin{array}{l}\text { Rude 9: It is obligatory that each Customer belongs to one and on } \\
\text { Customer Type }\end{array}$} \\
\hline
\end{tabular}

Figure 3. Sample SBVR vocabulary and rules.

SBVR - SBVR is a standard [6] developed by the $\mathrm{OMG}$ to facilitate formal documentation of the goals governing the operations of a business. Traditionally, businesses use operational guidelines defined across ad hoc documents lacking formal structure to document business goals. The SBVR specification provides formal semantics to enable consistent understanding and interpretation of the operating principles of businesses. SBVR is independent of information systems, and thus provides a means of bridging the gap that commonly exists between business operational guidelines and the systems that implement them. To facilitate the interchange of data and provide for standardized data interfaces between entities, artifacts, or tools, the SBVR meta-model generation process uses a structured, fact-oriented approach incorporating a formal business vocabulary and a set of business rules built on that vocabulary.

An extract of the SBVR metamodel for a distributed billing system is presented in Fig. 3 .

N3 - N3 [7] is a language designed to be a compact and readable alternative to RDF/XML. It is used in the DRM to model policies governing relationships in a technology-neutral manner. In addition to supporting the full expressiveness of the RDF, N3 also supports the encoding of RDF rules logic. An important aspect of N3 is the ability to explicitly specify hypothetical subgraphs for use in rules or to load graph subsections from other sources. In the context of this work, this approach can be exploited to define operational policy rules based on the contents of the knowledge base [8].

For example, the N3 snippet (Fig. 4) allows people from ABC_Corp working on the collabo- rative FAME project to access sales orders in $\mathrm{XYZ}$ Corp relating to the FAME consortium.

This example illustrates an interoperability challenge whereby different organizations represent information differently. In XYZ_Corp ontologies the FAME project is represented as an ontological instance, whereas in $A B C$ Corp a project is only a string value used to tag employees.

\section{TCBPMS}

The CBPMS [9] is a distributed policy management approach for federated systems. It utilizes a flexible, graph-based capability authority model to partition and delegate federated capabilities or services as delegation chains. The TCBPMS solution extends the basic CBPMS features with trust management functions that address threats from malformed or malicious federated principals, and provides increased flexibility in delegation chain reduction and local capability authority repartitioning. Dedicated policy logic supports secure decentralized reasoning within TCBPMS with an implementation based on public key certificates.

\section{Trust Management}

Network communications security technologies such as IPsec can be used to ensure secrecy, integrity, and authenticity of messages exchanged between domains. However, domains may make fraudulent statements over these secure channels about their capabilities or the capabilities of others. This threat may be due to inadvertent or malicious intent on the part of the domain's business process, or as a consequence of an intruder compromising part of the federationsupporting infrastructure (e.g., a policy server). Therefore, TCBPMS provides not just secure channels between domains, but also secure formation and management of relationships between the domains.

In centralized architectures it is relatively straightforward to provide this security infrastructure since all related policies, capabilities, and so on are centrally stored and managed on a secure host. However, as autonomous self-governing entities, domains should not have to rely on some central authority when forming and managing relationships. This domain self-determination is achieved by taking a trust management approach to distributing the LRM (the policies, capabilities, etc.) across the domains whereby federation policy decisions can be made locally in a domain without reference to any central authority. Trust management uses cryptographic certificates to implement this secure distribution and ensure that one domain cannot make fraudulent statements about the capabilities of another.

Capability Authority Models - Capability authority models describe how the capabilities shared by a domain are bundled together into sets of capabilities and specific associated permissions. These bundles are known as capability authorities. A fundamental aspect of the capability authority approach lies in the ability to compare two capability authorities and decide whether the first capability authority encapsu- 
lates the second according to the model. This allows capability authorities representing arbitrary aggregations of specific permissions to be distributed between federated domains. Whenever a third party invokes a capability, the federated domain merely establishes whether the capability being invoked is encapsulated by a capability authority that has been issued to that domain. In addition to maintaining the capability authority graph, it is necessary for the distributed TCBPMS instances to maintain a capability authority delegation graph that indicates where a given capability authority resides.

Capability models thus provide an access control mechanism across domain relationships. Capability authorities can also be associated with policy rules defined within the management system of the domain that controls the capabilities. This provides a flexible and expressive means of applying access control to capability sharing in federal relationships without requiring that all parties support common policy or information models.

In addition to capability discovery and description, infrastructure is required to enforce security requirements - so that exposed capabilities can only be used by those parties with whom they been shared and that this use does not inadvertently expose any information which might help hostile parties from gaining access to confidential information. Additionally, it is generally desirable to limit the amount of internal information that is exposed to third parties to the absolutely minimum necessary.

To tackle these problems, the TCBPMS provides a trusted capability authority architecture which aims to fully insulate the exposed capabilities from internal processes. The trusted capability authority layer cryptographically signs each shared capability authority when it is distributed to third parties. When a third party attempts to use the capability, the signature is checked to ensure that the specific capability being used has been shared with that third party. Arbitrary repackaging of signed, shared authorities is supported as this helps with manipulation by different domains in terms of their local policy or resource models.

\section{TOOL CHAIN FOR Automatic Generation of TMs}

TMs extend the understanding of context using a graph to document the relationships of software artifacts (business goals, policies, contracts, and processes) throughout the life cycle of software [10]. The TMs for each type of software artifact can be combined to form an intradomain TM documenting the relationships between all software artifacts. In a manner similar to the construction of intradomain TMs, a TM can be constructed for each interdomain relationship and combined with the intradomain maps, resulting in a full solution TM. The automatic generation of TMs is realized using a model-driven approach and a number of domain-specific languages (DSLs). We use the DEN-ng information model as the foundation, specifically the policy model, combined with a contract model based on TeleManagement Forum TMF 053b. In a

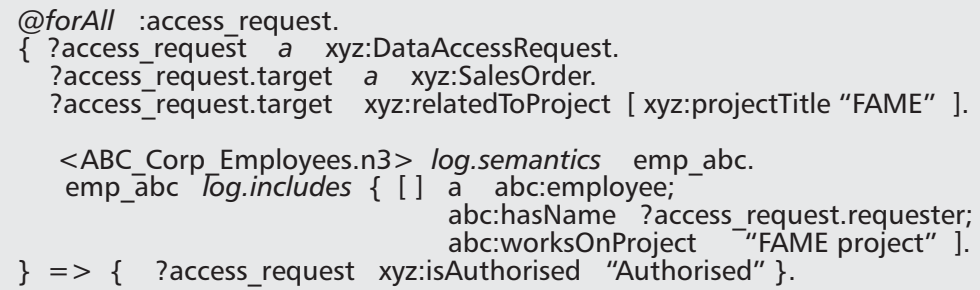

Figure 4. Sample N3 rule.

manual step we define a stratified policy language and a contract language (EBNF), which in turn provides the basis for developing a tool chain to apply them to generate TMs. The policy language represents a high-level language following the classification of the DEN-ng policy model and provides dialects to address the different needs of various constituencies (i.e., business analyst and system administrator). The contract language represents a formal definition for contract-based components. Both languages allow a relationship to be defined with the contract part specifying the agreed conditions and rules of the relationship and the policy part specifying the control of the relationship, for instance, in cases where contracts are violated.

Figure 5 shows the implemented tool chain that supports the automatic generation of TMs. The policy language is named DPOL, and the contract language is named L-ADS. We have developed two different set of tools, one based on xTeXT (Eclipse model-based development environment) and one based on ANTLR (a parser generator). With xText, we can generate editors as Eclipse plug-ins and then hand over the resulting policy and contract specifications to an ANTLR compiler, which transforms between different textual representations. Finally, we can populate our (DEN-ng) knowledge base with all information needed to generate TMs (mid-right part of the figure). The knowledge base can handle intra- as well as interdomain maps. We also have developed a set of clients (Java Policy client, Conflict Detection) to analyze the generated maps.

\section{USE CASE: Service Billing as a Service}

In order to illustrate the advantages of our approach to building multidomain IT systems we provide this use case for a key SDP enabler - a comprehensive billing function.

\section{Service Structure}

Figure 6 illustrates a billing function that is itself a multidomain service which includes third-party components serving a number of CSPs to provide a unified billing solution (one of the distinguishing features of CSP networks). In a next-generation network the number of domains traversed for service delivery increases, and flexible billing architectures make it more likely that CSPs will be able to appropriately meet the varied needs of customers. Hence, it is natural to allow outsourcing of specialized expertise in specific aspects of the billing flow. The disadvantage 


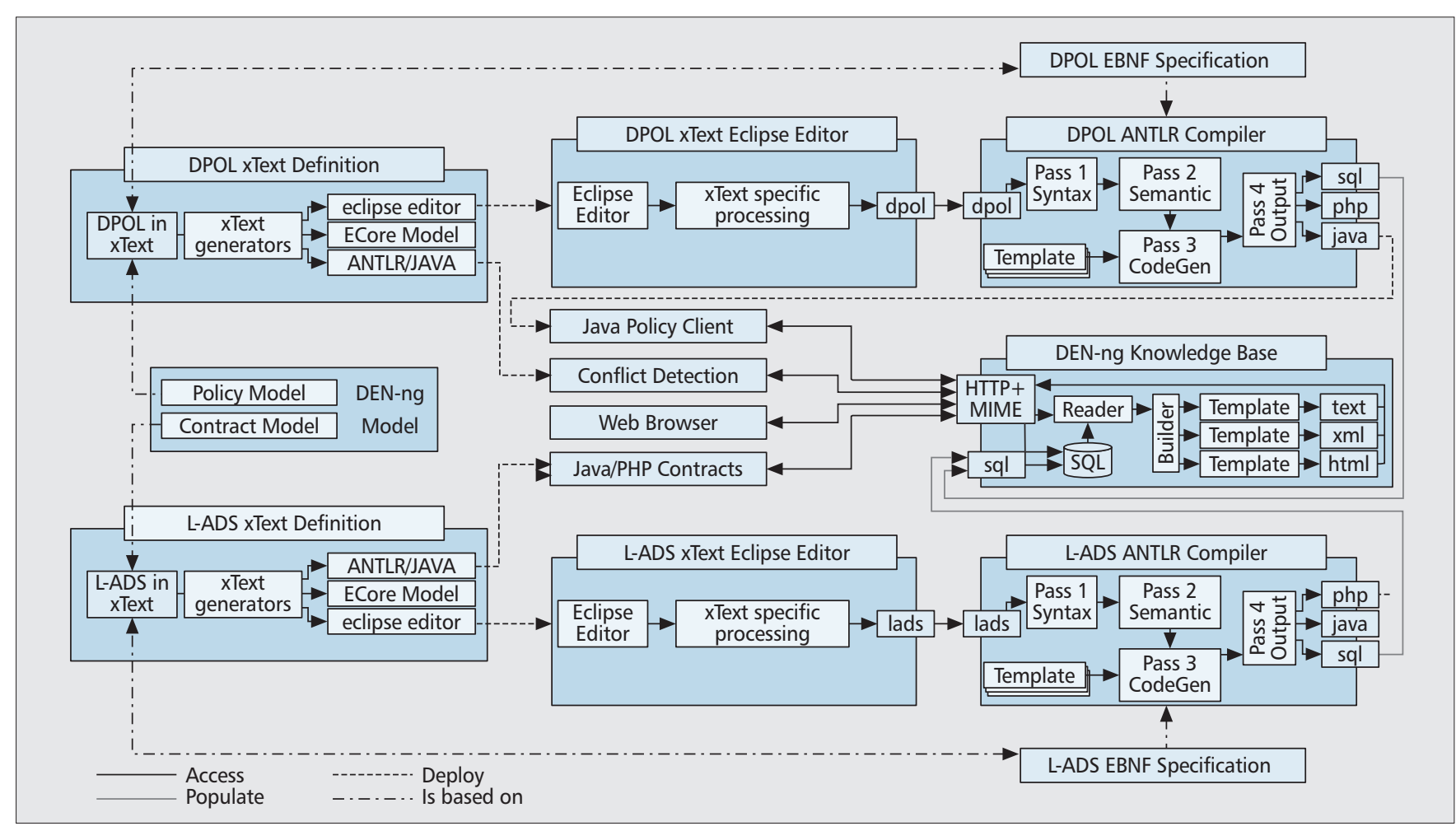

Figure 5. Traceablilty map tool chain.

of this approach for a traditional IT architecture is that as the number of relationships or integration points grows, the more likely the system is to become brittle and inflexible since each external and internal relationship must be maintained by custom gateways. In our approach formal models of the relationships keep the integration points lightweight, adaptable, and explicitly manageable because the relationship models allow automated or semi-automated negotiation and renegotiation of the interface capabilities, and the mappings between the exported or imported capabilities and internal policies and models.

\section{DYNAMIC BEHAVIOR}

The subsections below discuss the applicability of our domain relationship manager architecture to the use case.

Domain Matching in Action - Emerging billing environments may use different suppliers to support presentation of intermediate or final bills to the customers: third-party presentation vendors are selected on the fly based on customer needs (cost, availability, performance, type of bill presentation requested), context (location, time), or many other criteria derived from the business strategy of the bill provider and the desires of the bill consumer. In such an environment it is essential that, rather than longterm static interdomain bindings that tend to be a compromise for the billing provider and an average customer (and thus not meeting the business goals and strategy of either party), interdomain relationships are dynamic and flexible to allow the best available fit between specific customers' desires and the capabilities of the billing presentation service providers.
Capability Discovery - To negotiate sharing regimes between domains, the parties must support discovering, reasoning and negotiating about the capabilities other parties could make available. Fully automating this discovery and negotiation is an extremely difficult problem because the nature of negotiation is such that even revealing which capabilities are available for sharing may be valuable information that could compromise a party's bargaining position. Therefore, we assume that the parties to such federations have pre-existing legal relationships and are already in a situation where they know which third-party capabilities they wish to utilize. This assumption allows us to concentrate on the problem of relationship participants translating the capability authorities they have been delegated into sets of capabilities they can invoke. Our approach is to provide semantically rich descriptions of capability authorities, using the RDF to describe the semantics of the capabilities being shared and the set of permissions they encompass. This allows third parties who have been delegated particular capability authorities to use SPARQL-based queries to translate their capability authorities into concrete capabilities that they can directly invoke.

Establishing Trusted Relationships - A customer trusts billing from its CSP, which in turn federates with a third-party billing presentation service by signing a capability (certificate) that delegates authority on billing to the third party. This certificate provides unforgeable proof to the customer that it can trust billing information from the third party without having to interact with the CSP. This is a simple scenario; in practice, certificates encode the policies, capabilities, 
and so on across the LRM that are related to federations involving this third party.

\section{CONCLUSIONS}

We have presented a comprehensive model and a novel architecture for managing relationships between the adaptive domains making up nextgeneration CSP IT architectures. In the past management of interdomain relationships has only focused on centralized and static solutions. In practice, this has produced brittle, limited, expensive, and non-standard SDP integrations. Even when dynamic aspects were considered (e.g., with trader-style matchmaking services), the focus was on establishing the appropriate relationship rather than managing and maintaining a relationship through its life cycle. As business models and service production become more flexible, the importance of supporting this dynamic approach will increase.

\section{ACKNOWLEDGMENT}

This research is partially supported by the Science Foundation Ireland (Grant 08/SRC/I1403); see http://www.fame.ie.

\section{REFERENCES}

[1] D. Cannon and D. Wheeldon, "ITIL Service Operation," The Stationery Office, 2007.

[2] R. Brennan et al., "Policy-Based Integration of Multiprovider Digital Home Services," IEEE Network, vol. 23, no. 6, Nov. 2009, pp. 50-55.

[3] J. J. Fleck II, "Next Generation Management for Adaptive Environments," NOMS, Osaka, Japan, Apr. 2010.

[4] G. Klyne, J. J. Carroll, and B. McBride, Eds., "Resource Description Framework (RDF): Concepts and Abstract Syntax" W3C, Feb. 10, 2004.

[5] OWL Working Group, "OWL 2 Web Ontology Language Document Overview," W3C, Oct. 27, 2009.

[6] Object Management Group, "Semantics of Business Vocabulary and Business Rules (SBVR)," v. 1.0, 2008.

[7] Tim Berners-Lee (Ed.), "Notation $3-$ A Readable Language for Data on the Web," W3C, 2006; http://www.w3.org/Designlssues/Notation3.html

[8] L. Kagal, "A Policy-Based Approach to Governing Autonomous Behavior in Distributed Environments," Ph.D. thesis, Univ. MD Baltimore County, Sept. 2004.

[9] K. Feeney, D. Lewis, and D. O'Sullivan, "Service Oriented Policy Management for Web-Application Frameworks," IEEE Internet Comp., vol. 13, no. 6, Nov./Dec. 2009, pp. 39-47.

[10] S. van der Meer and J. Fleck II,"Traceability Maps as a Conceptual Tool for Managing Software Artifacts," 15th HP Software Univ. Assn., Marrakech, Morocco, June 22-25, 2008.

\section{BIOGRAPHIES}

ROB BRENNAN (rob.brennan@cs.tcd.ie) is a research fellow in the knowledge and data engineering group (KDEG), Trinity College Dublin (TCD), Ireland. His research interests include semantic interoperability, intelligent distributed systems, and the application of linked data to systems management. He has contributed to 3GPP, TMF, IETF, and OMG communications standards. He has a Ph.D. (2004) from Dublin City University. Prior to TCD he worked in the Ericsson network management research center and a number of startups.

KEVIN FEENEY (kevin.feeney@cs.tcd.ie) is a research fellow in the Knowledge and Data Engineering Group in the School of Computer Science and Statistics at TCD. His research interests include inter-organizational management and

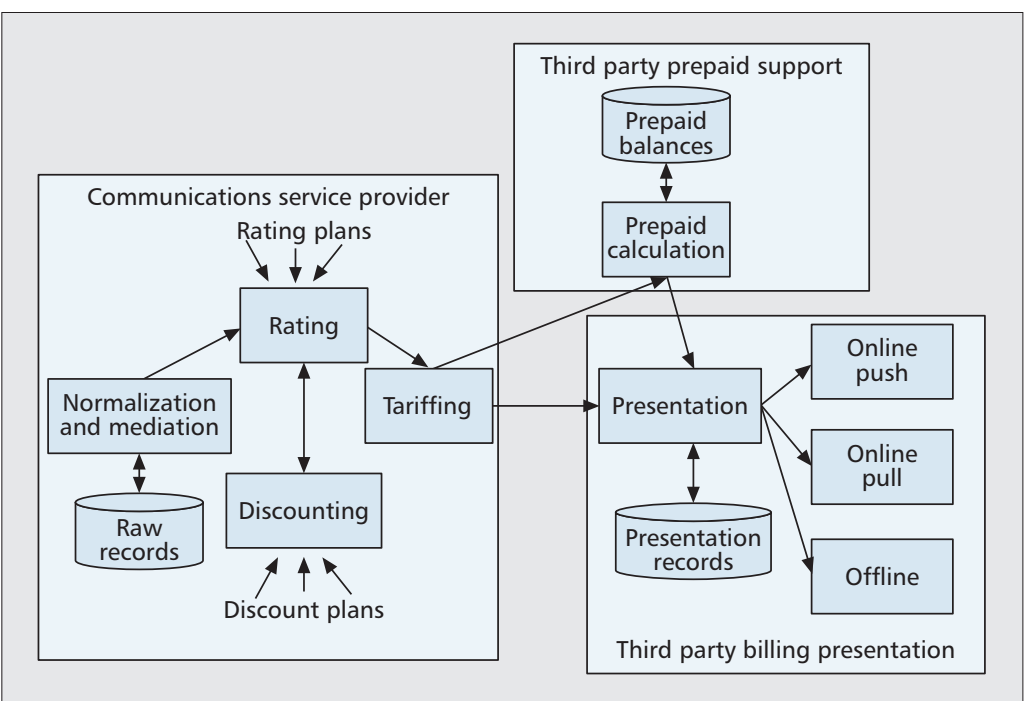

Figure 6. Use case: billing domain service structure.

applying collective intelligence to management problems. He has a Ph.D. in computer science from TCD.

JOHN KEENEY (john.keeney@cs.tcd.ie )is a post-doctoral researcher in the Knowledge and Data Engineering Group in TCD. His research focuses on the management of autonomic adaptable systems, particularly networking and telecom systems. He addresses the practical application of semantic web technologies and other knowledge-based techniques in the domains of self-adaptive systems, policy engineering, personalization of adaptive systems, visualizing the state and constraints of managed systems, and event-based middleware.

DECLAN O'SUlLIVAN (declan.osullivan@cs.tcd.ie) has a B.A (Mod), M.Sc., and Ph.D., all in computer science, from TCD. In addition, he has worked in industry for 13 years. His research interest lies in the identification and development of techniques to enable semantic mapping as a means to enhance collaboration in heterogeneous environments.

JOEL J. FLECK II (joel.fleck@hp.com) is chief architect in the Software and Solutions CTO organization, HewlettPackard's Office of Strategy and Technology with a focus on architectures for adaptive management of distributed systems. Previously, he spent 16 years at Bell Laboratories and Bell Communications Research. He graduated from the University of Michigan with an M.S. in industrial and operations engineering, and the University of Vermont with a B.S. in computer science. He is a Distinguished Fellow of the TeleManagement Forum.

SIMON FOLEY (s.foley@cs.ucc.ie) is a statutory lecturer at University College Cork where he teaches and conducts research in computer security. He serves on the editorial board of the Journal of Computer Security and has served as Program Chair of the IEEE Computer Security Foundations Workshop and the ACSAC New Security Paradigms Workshop. He has over 70 international peer-reviewed publications on security, and his research interests include trust and risk management, security modeling, and security psychology.

SVEN VAN DER MEER [M] (vdmeer@ieee.org) received his degrees (Diplom [1996], Ph.D. [2002]) from Technical University Berlin, Germany. He joined Waterford Institute of Technology in 2002, where he is currently a senior research fellow for network and service management. Most of his current time is dedicated to autonomic management as technical leader of Irish and European research programs developing strong links with industrial partners (Cisco, Ericsson, and HP). 